Classification

Physics Abstracts

61.16Di

\title{
Scanning tunneling microscopy study of a DNA fragment of known size and sequence
}

\author{
Monique Marilley $\left({ }^{1}\right)$, Philippe Pasero $\left({ }^{1}\right)$, Alain Humbert $\left({ }^{2}\right)$, Samuel Granjeaud $\left({ }^{2}\right)$, Michel \\ Dayez $\left({ }^{2}\right)$, Roger Pierrisnard $\left({ }^{2}\right)$ and Bertrand Jordan $\left({ }^{3}\right)$ \\ (1) Laboratoire de Génétique, URA CNRS 1189, 27, Bd J. Moulin, 13385 Marseille Cedex 5, France \\ ( ${ }^{2}$ ) Laboratoire de Physique des Etats Condensés, URA CNRS 783, Parc Scientifique et Technologi- \\ que de Luminy, Case 901, 13288 Marseille Cedex 9, France \\ $\left({ }^{3}\right)$ Centre d'Immunologie de Marseille-Luminy, Parc Scientifique et Technologique de Luminy, Case \\ 906, 13288 Marseille Cedex 9, France
}

(Received 19 October, 1993; accepted 17 November, 1993)

\begin{abstract}
Résumé. - Des essais pour visualiser un fragment d'ADN qui puisse servir de test pour des expériences ultérieures ont été réalisés en microscopie par effet tunnel (STM). Nous présentons des observations faites sur un fragment de restriction de $350 \mathrm{pb}$ provenant de pBR322, déposé sur la surface d'un monocristal naturel de graphite et imagé sous vide. La mesure de la longueur et des paramètres hélicoïdaux de la molécule s'ajoutant au fait qu'aucun aspect du substrat ou des caractéristiques électriques ne suggérait un artefact du graphite, ont permis une identification non ambiguë de la molécule. Limage de la molécule complète d'ADN a été obtenue et des détails en haute résolution sont montrés. Leur correspondance avec des séquences d'ADN précises peut être déduite de leur localisation sur l'image puisque la séquence et l'orientation de la molécule sont connues. Les variations générales de même que les variations locales des caractéristiques structurales le long de la molécule d'ADN sont donc maintenant accessibles à l'analyse par imagerie STM. La capacité du STM à analyser les structures d'ADN impliquées dans l'activité génique apparaît ainsi très prometteuse.
\end{abstract}

\footnotetext{
Abstract . - Assays to visualize a DNA fragment that may serve as DNA test for further experiments were performed by scanning tunneling microscopy (STM). We present observations on a 350 bp restriction fragment from pBR322 deposited on natural graphite single crystal surfaces and imaged in vacuum. Measurement of the length and of helical parameters of the molecule in conjunction with the fact that no features of the substrate or electrical characteristics were suggestive of a graphite artifact, allowed unambiguous identification of the molecule. Imaging of the entire DNA molecule has been achieved and high, resolution details are shown. Their correspondence to precise DNA sequences may be deduced from their localization on the image since the DNA sequence and orientation of the molecule is known. Therefore, both global and local sequence-directed variations of structural features along a DNA molecule are now accessible to analysis with STM imaging. The ability of STM to investigate DNA structures involved in gene activity appears thus to be very promising.
} 


\section{Introduction.}

Study of DNA sequences involved in gene regulation has proven to be a powerfull approach in genetic studies. However there is now growing evidence that not only sequences but structural characteristics associated to these DNA regions have to be taken into account for making further progress in the understanding of gene function [1,2].

In that sense, the use of scanning tunneling microscopy (STM) to analyze structural features of DNA might reveal to be a major application of this microscopy and would open a new field of investigation in genetics. It is in this context that we present our first attempts to visualize a DNA fragment.

Scanning tunneling microscopy (STM) has proven to be a powerful method to obtain highresolution images of uncoated biological molecules in vacuum, air or liquid environments [3-7]. Atomic-resolution images of double-stranded DNA have been obtained in ultra-high vacuum [8]. However, STM imaging of naked biological molecules is not a routine process yet. The poor reproducibility of the experiments observed up to now is due mainly to lack of reliable methods able to limit motion of the molecules under the scanning tip during STM imaging. This explains why most recent developments are mainly dealing with methods likely to improve adhesion of the molecules to their support [9]. These methods are now making progress, however, treatments used to allow sticking of molecules may also affect their structure. An alternative approach is to minimize the interaction forces between tip and sample. Therefore, we have developed a STM apparatus which allows the use of very small currents $(<1 \mathrm{pA}$ in routine experiments, which is about a hundred time lower than generally used currents), and make possible the visualization of objects that are poorly conductive or badly- coupled to the substrate.

Attempt to visualize DNA molecules at atomic scale resolution constitutes another very promising axis of investigation. However, due to data acquisition limitations, a very high resolution has only been obtained on very short parts of DNA molecules, so that the location of the region imaged and therefore its sequence are not known [8]. It is worth pointing out that both macroscopic and local deflections of the helix axis play an important role in protein-DNA interactions and are involved in fundamental genetic processes [10-13]. Long-range domains of altered DNA structures, corresponding to sequence-directed curvatures of the helix axis, have been extensively studied over the past few years $[14,15]$. Numerous convenient techniques have been employed for studying these global alterations. However, local alterations of the helix affecting the size and the shape of grooves are mainly addressed through very cumbersome methods [11]. Results already obtained by STM imaging of DNA $[8,16]$ strongly indicate the potential of STM microscopy for investigating these DNA structures. However, obtaining reliable information on a DNA molecule of biological interest requires not only to perform high-resolution imaging, but also to be able to identify and to localize the analyzed regions along the molecule.

In this paper, we present STM observations of double stranded DNA molecules (pBR322 restriction fragment) in vacuum. We have chosen to study a small DNA molecule of known size and sequence. High-resolution imaging, compatible with visualization of the complete molecule, was achieved. Determination of the average parameters of the double helix was performed, showing that all the observed structures are consistent with known characteristics of the molecule studied. Moreover, detailed observations are reported, that can be localized on the molecule. These results strongly indicate the potential of STM microscopy for the investigation of DNA structures involved in gene activity. 


\section{Results and discussion.}

The scanning tunneling microscope used for these studies was designed by our group and previously used to image small metallic clusters deposited under ultra-high vacuum (UHV) on graphite surfaces [17-19]. It is mounted inside a UHV chamber with base pressure of about $10^{-10}$ torr, but the DNA molecules were imaged in a vacuum of $10^{-7}$ torr, as in electron microscopy (EM) studies. Atomic resolution is routinely achieved with this microscope on flat single crystal surfaces or three-dimensional (3D) clusters [19]. It allows the use of very small tunneling currents $(<1 \mathrm{pA})$.

Cleaved Highly-Oriented Pyrolitic Graphite (HOPG) and gold (111) surfaces have been extensively used for STM study of biological molecules [9]. As far as STM imaging is concerned, HOPG appears to be a good substrate because of its mechanical rigidity, good electrical conductivity and flatness at the atomic scale. However, some features observed on graphite, such as steps and/or domain walls have led sometimes to misinterpretations [20]. Fortunately, careful analysis of the geometry of these features makes possible the distinction between biological molecules and artifacts [21]. Figure 1 shows strand-like structures generated by cleavage of natural single crystal graphite sample. The twisted appearence of these structures can lead to misinterpretation. But it also appears clearly that other parameters differ significantly from those of DNA molecules. This is also the case for all the other described artifacts on HOPG. The different types of strand-like structures that can be present on freshly cleaved HOPG have been cataloged and characterized by Chang and Bard [22]. However, none of these confusing structures display simultaneously all the helical parameters of DNA molecules (i.e. helix sense, helix pitch, width, height, ... ). Moreover, in contrast to biological specimens, these artifacts are characterized by high conductivity and general orientation along crystallographic directions.

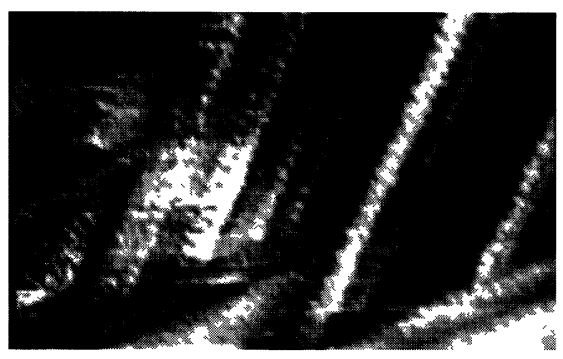

Fig. 1. - STM image of graphite strand-like structures. This region was selected because it contains a high concentration of defects and allows then statistical analysis. Here, most of these surface features present a clear left-handed helicity. The average periodicity was determined to be $9 \pm 0.5 \AA$. The dispersion of the mean helical pitch is similar between different graphite fibers and along a given one. In contrast, the average width varies significantly between fibers $(16 \pm 6 \AA)$. These structures are straight and make sharp $120^{\circ}$ angles with one another, revealing their orientation along cristallographic directions of graphite. This image measures $347 \AA$ by $220 \AA$.

In this work, natural graphite single crystals cleaved in air with a razor blade as a wedge were used as substrates for the DNA molecules instead of HOPG since our previous experiments on the nucleation of small metallic particles have shown that such surfaces exhibit a much lower density of defects, so that much of the problem may be prevented.

Since length is an additional criterion for identifying the molecule, we have chosen to work 
on a DNA restriction fragment of known size. The $350 \mathrm{bp}$ pBR322 restriction fragment (HindIII$B a m \mathrm{HI}$ ) corresponding to the 5' region of the tet ${ }^{R}$ gene has been analyzed. The choice of the DNA molecule was carefully undertaken, having in mind that we need to possess the greatest amount of information on the molecule. In addition to general X-ray crystallography information, these data include the complete nucleotide sequence of the molecule and various studies on higherorder DNA structure. The latter concerned overall helix axis bending studied by polyacrylamide gel electrophoresis analysis of DNA mobility [23], direct EM observation of variation of local curvature along the molecule [24] and comparison with theoretical curvature diagrams calculated for pBR322 [25]. In addition to general information on DNA structure, the choice of the well studied DNA molecule pBR322 provides us with another set of criteria for identification and allows comparison with E.M. data. This confirmation by other techniques is a necessary step at the present phase of development of STM.

Figure 2 shows a STM image of a complete DNA restriction fragment lying on a flat surface. Its trajectory is neither straight nor aligned along crystallographic directions of the graphite surface and does not cross graphite steps, as it is the case for meandering strand-like artifacts. The molecule appears to be slightly broadened when it is perpendicular to the fast scanning direction (horizontal axis). This observation suggests that the interaction forces between the probe tip and the molecule are slightly increased for that orientation. However, these forces remained weak, as is evidenced by the fact that we were able to image continuously the molecule during three days without obvious modification of the STM image. Several interpolated cross-sections perpendicular to the molecule axis are shown in figure 3 . They confirm that the molecule is lying on a flat part of the substrate. Its measured length ( $888 \AA$ ) fits to the expected length for a $350 \mathrm{bp}$ DNA molecule, well within the accuracy of the calibration of the probe tip piezo scanner $( \pm 5 \%)$ (E.M.measurement of the same molecule is $1015 \pm 160 \AA$ ) The average width of the molecule was found to be $26.2 \AA$. The right-handed helix is clearly visible on the figure. In order to get quantitative values for the helix parameters, a spectral analysis of the power density of the image has been done from the bidimensional Fourier transform. We thus determined an average helix pitch value of $24.8 \AA$ and an average angle between the grooves and the helix axis of 68.7 . These values appear more closely related to the conventional A form determined by X-ray crystallography than to B-DNA (see Tab. I). This result is consistent with previous EM, AFM and STM studies (26, $27,8)$ and is likely to be due to partial dehydratation of the molecule.

In figure 2, remarkable variations of the overall path of the double-helix are observed. The trajectory of the helix axis appears asymmetrically curved, presenting one major bent region in the right side of the figure. We have compared the STM experimental curvature map to those previously reported from electron microscopy analysis and theoretical calculations for pBR322. Electron microscopy data and three out of the four theoretical models give strikingly similar results [25]. It is worthwhile to mention that the overall path of the STM imaged DNA fragment, as well as the position of bending elements along the molecule (Fig. 4), are consistent with these data.

Thus, comparison of EM data, theoretical data and STM experimental values shows a complete agreement. The DNA helix trajectory recorded by STM imaging corresponds to the one expected for this molecule (Fig. 2). It is thus appearent that we are looking at a structure which presents meanders having the same amplitude and location as bending elements of the restriction fragment under study. This result, beside length, helical parameters and conductivity characteristics give additional evidence that the structure observed corresponds to the pBR322 restriction fragment. Moreover, it is worthwhile to note that beside giving a new element for the identification of the structure this result indicates strongly that global and local variations of the helix axes might be investigated with this new technology. 


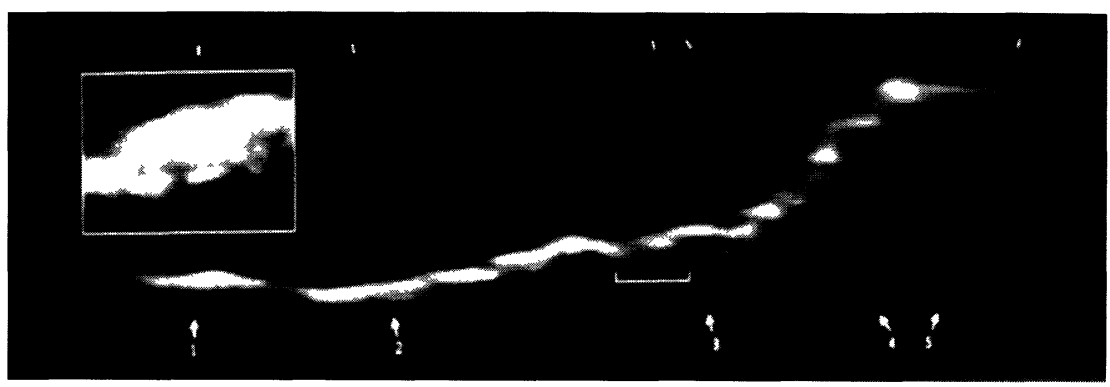

Fig. 2. - STM image (512 points per line, 140 lines) of an isolated DNA molecule corresponding to a 350 bp pBR322 restriction fragment (HindIII-BamHI). A $2 \mu \mathrm{l}$ drop containing $20 \mathrm{ng}$ DNA was deposited at $4{ }^{\circ} \mathrm{C}$ on a freshly cleaved natural graphite single crystal surface in air and dried in vacuum. DNA molecules were imaged in a vacuum of $10^{-7}$ torr. The images were recorded in the constant-current mode, which allows quantitative height measurements and can be used to track surfaces that are not flat at the atomic level. Slow scan rates (100-300 $\AA / \mathrm{s})$ were used in order to minimize friction between the probe tip and the DNA molecules. Typical tunneling voltages were 0.1 to $2.0 \mathrm{~V}$. The probe tips were tungsten threads electrochemically-etched in $2 \mathrm{~N} \mathrm{NaOH}$ solution. They were systematically cleaned in- situ by tunneling over clean surfaces (gold, graphite) before any imaging of DNA molecules. An enlarged portion of the image (bracket) is shown in the insert. Arrows indicate the position and direction of the cross-sections shown in figure 3.

Table I. - Comparison of DNA dimensions derived from STM observations and X-Ray crystallography data.

\begin{tabular}{|c|c|c|c|c|}
\hline Environment & STM 1 & STM 2 & $\begin{array}{c}\text { X-ray 3 } \\
\text { (A-DNA) }\end{array}$ & $\begin{array}{c}\text { X-ray 4 } \\
\text { (B-DNA) }\end{array}$ \\
\hline Helix sense & right & right & right & right \\
\hline Helix pitch & $24.8 \AA$ & $36 \AA$ & $24.6 \AA$ & $33.2 \AA$ \\
\hline Molecular width & $26.2 \AA$ & - & $25.5 \AA$ & $23.7 \AA$ \\
\hline Major groove & $\begin{array}{c}\text { Deep and } \\
\text { narrow }\end{array}$ & Wide & Deep and narrow & $\begin{array}{c}\text { Wide and } \\
\text { moderately deep }\end{array}$ \\
\hline Minor groove & $\begin{array}{c}\text { Wide and } \\
\text { shallow }\end{array}$ & Narrow & $\begin{array}{c}\text { Wide and } \\
\text { shallow }\end{array}$ & $\begin{array}{c}\text { Narrow and } \\
\text { moderately deep }\end{array}$ \\
\hline
\end{tabular}

1. average values from this report - 2. Lindsay et al., 1989 (31) - 3. Conner et al., 1981(32) - 4.

Drew et al., 1981(33). 


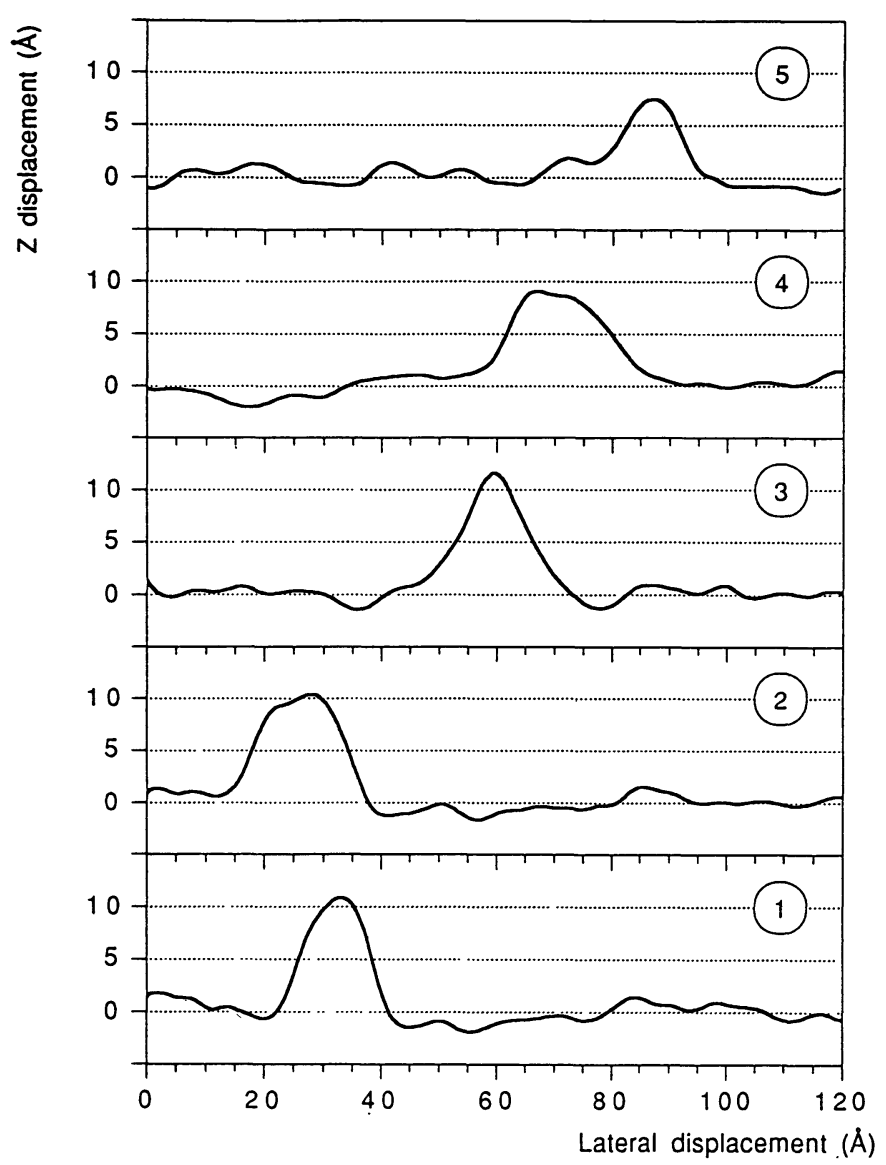

Fig. 3. - Interpolated cross-sections perpendicular to the molecule axis corresponding to the arrows in figure 2. The sections were chosen as representative of different regions of the molecule. These profiles confirm that the molecule is lying on a flat part of the substrate.

Figure 5 compares a van der Waals space-filling model for A-DNA (Fig. 5a) calculated from crystallographic data and the enlarged region of the molecule shown in the insert (Fig. 2). The most striking feature is the good correspondence observed between the experimental and theoretical model outlines. Some structures within the molecule can also be distinguished. However, the experimental resolution of figure $5 \mathrm{~b}$ is not sufficient to resolve the DNA bases. This results mainly from the fact that this figure is extracted from the image of the whole DNA fragment shown in figure 2 which was scanned with 512 sampling points per line. Thus, the sampling steps are 2.74 $\AA$ parallel to the fast scan direction and $1.37 \AA$ perpendicular to it. The ultimate resolution of the microscope is expected to be much better.

An asymmetry of the STM image of the DNA molecule is also visible in figure 5b. On the right side of the molecule, the outline appears to be more strongly corrugated than on its left side. This may result from an asymmetric shape of the probe tip. As a matter of fact, the effect is visible in figure 2 all along the DNA fragment.

In figure $5 \mathrm{c}$, the left half of the van der Waals model has been juxtaposed to the right half of the experimental STM image. It is striking to see how good the correspondence is between the 




Fig. 4. - Comparison of the experimental curvature map with a theoretical diagram of curvature for the corresponding restriction fragment. Three dimensional coordinates of the helical axis are calculated along the sequence using parameters of the dinucleotide wedge model [29]. Local curvature is determined within a window of $40 \mathrm{bp}$ moving along the DNA by $5 \mathrm{bp}$ segment steps and calculated according to Muzard et al. (25). The positions of maximum and minimum curvature are seen here to be very similar for both curves. A strong bending element predicted from the modelisation at the 5' terminal end is not observed for the STM imaged molecule. Since this element is located very close to the cutting site, it might be relaxed following linearization of the molecule.

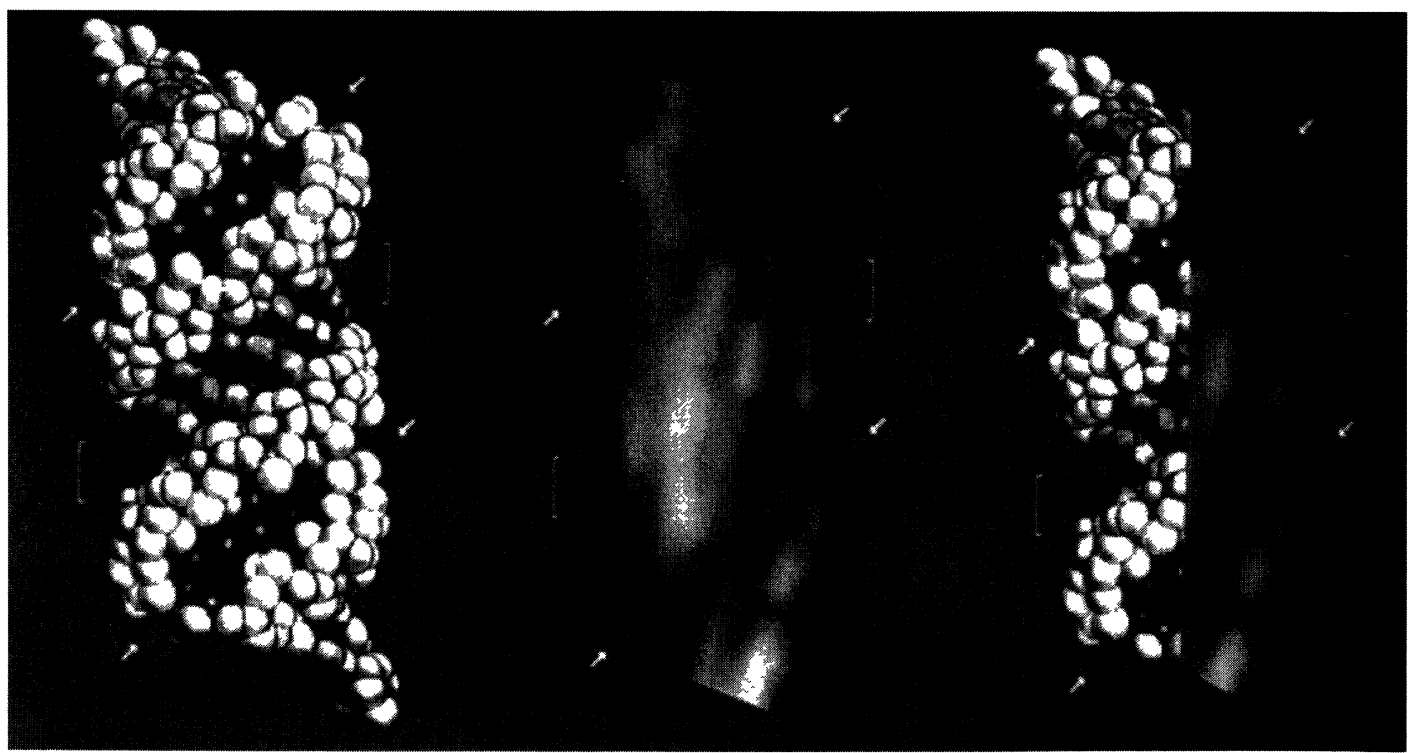

Fig. 5. - Comparison between the enlarged portion of the molecule shown in the insert of figure 2 and a text book model for A-DNA (van der Waals space-filling model) calculated from X-ray crystallographic data [30]. The STM image shows two helix turns. The deep and narrow major groove is clearly visible (arrows), as well as characteristic features associated with the wide and shallow minor groove (brackets). c. The left half of the van der Waals model has been juxtaposed to the right half of the experimental STM image to demonstrate the observed good agreement between them. 
characteristic supra-atomic features on both sides. This result shows that STM has potential for high-resolution observations of DNA features. Very rapid progress can therefore be expected from STM studies of DNA binding sites involved in protein interaction.

\section{Conclusion.}

STM observations of double-stranded DNA restriction fragments of known length and nucleotide sequence are shown. We observed that all the parameters of the molecule: right- handed helicity, helix pitch, length, width, height and groove shape are fully consistent with published crystallographic data and previous atomic-resolution STM experiments on double- stranded DNA $[8,16]$. Moreover, this sample lies on an atomically flat surface and the characteristics of conductivity are those of biological molecules (poorly conductive samples). Taken together, these features rule out the possibility of misinterpretation and allow an unambiguous identification of the DNA molecule.

Visualization of local and global sequence-directed structural features is also reported. Results concerning the variation of the curvature along the DNA molecule are in good agreement with previously published EM studies, as well as with theoretical curvature maps [25] Since bendings observed on the molecule correspond as well for their localisation as for their amplitude with experimental and theoretical values and since they were also observed on other molecules (two incomplete fragments that were neglected because the length criterion could not be used), these bendings are not likely to be caused by local interaction betwen DNA and graphite. They appear, then, to represent natural curvature. This shows that using STM, intrinsic curvatures of DNA can be studied at the nanometer scale, although present state of the art has limited the analysis to a very small number of molecules.

Our STM studies have not yet been performed at atomic resolution. However, the observed stability of the isolated molecules on their support suggests that such studies can be done in a reproducible way. Although improvements in sample preparation are necessary [9], STM highresolution imaging is clearly a promising way to study DNA structures involved in gene activity [28]. It is now clear that sequence-dependent DNA curvature governs the accessibility of bases to DNA-binding proteins by determining their outwards or inwards orientation [19]. A main contribution of STM to nucleic acids research may be to allow the study of the intimate structure of binding sites within their general DNA structural environment.

\section{Acknowledgements.}

This work was supported by research grants from l'Association Française de lutte contre les Myopathies, le Conseil Général des Bouches-du Rhône, le Conseil Régional PACA, la Mairie de Marseille, la Ligue Nationale Française Contre le Cancer.

\section{References}

[1] Calladine C.R. and Drew H.R., Understanding DNA. The molecule and how it works. Academic Press Inc. (1992). 
[2] Hagerman P.J., Sequence-directed curvature of DNA, Ann. Rev. Biochem. 59 (1990) 755-781.

[3] Engel A., Biological applications of scanning probe microscopes, Ann. Rev. Biophys. Biophys. Chem. 20 (1991) 79-108.

[4] Lilley D.M., Scanning tunneling microscopy of DNA, BioEssays 12 (1990) 131-132.

[5] Travaglini G., Imaging and conductivity of biological and organic material, Scanning Tunneling Microscopy Related Methods, NATO-ASI Series E184 (1990) 335.

[6] Descouts P. \& Siegenthaler H., 10 Years of STM. Proceedings of the Sixth Intern. Conf. and Scanning Tunneling Microscopy, Interlaken (12-16 August, 1991) North Holland (1992).

[7] Dunlap D. \& Bustamante C., Images of single-stranded nucleic acids by scanning tunnelling microscopy, Nature 342 (1989) 204-206.

[8] Driscoll R.J., Youngquist M.G. \& Baldeschwieler J.D., Atomic-scale imaging of DNA using scanning tunnelling microscopy, Nature 346 (1990) 294-296.

[9] Allison D.P., Bottomley L.A., Thundat T., Brown G.M., Woychik R.P., Schrick J.J., Jacobson K.B. \& Warmack R.J.,Immobilization of DNA for scanning probe microscopy, Proc. Natl. Acad. Sci. USA 89 (1992) 10129-10133.

[10] Eckdahl T.T. \& Anderson J.N., Conserved DNA structures in origins of replication, Nucleic Acids Res. 18 (1990) 1609-1612.

[11] Hagerman P.J., Straightening out the bends in curved DNA, Biochem. Biophys. Acta 1131 (1992) $125-$ 132.

[12] Travers A.A., Why bend DNA? Cell 60 (1990) 177-180.

[13] Travers A.A., DNA conformation and configuration in protein-DNA complexes, Curr. Op. Struct. Biol. 2 (1992) 71-77.

[14] Crothers D.M., Haran T.E. \& Nadeau J.G., Intrisically bent DNA, J. Biol. Chem. 13 (1990) 7093-7096.

[15] Trifonov E.N., DNA in profile, Trends Biochem. Sci. 16 (1991) 467-470.

[16] Youngquist M.G., Driscoll R.J., Coley T.R., Goddard W.A. \& Baldeschwieler J.D., Scanning-tunneling microscopy of DNA : atom-resolved imaging, general observations and possible contrast mechanism, J. Vac. Sci. Technol. B9 (1991) 1304.

[17] Humbert A., Pierrisnard R., Sangay S., Chapon C., Henry C.R. \& Claeys C., Morphological studies of small three-dimensional gold clusters on graphite by scanning tunneling microscopy, Europhys. Lett. 10 (1989) 533.

[18] Humbert A., Dayez M., Sangay S., Chapon C. and Henry C.R., Scanning tunneling microscopy and transmission electron microscopy studies of $\mathrm{Au}$ and Pd clusters grown on a clean graphite surface, J. Vac. Sci. Technol. A8 (1990) 311.

[19] Humbert A., Dayez M., Granjeaud S., Ricci P., Chapon C. and Henry C.R., Ultrahigh vacuum and air observations of Pd clusters grown on clean graphite, J. Vac. Technol. B9 (1991) 804.

[20] Clemmer C.R. \& Beebe T.P. Jr., Graphite: a mimic for DNA and other biomolecules in scanning tunneling microscope studies, Science 151 (1991) 640-642.

[21] Heckl W.M. \& Binnig G., Domain walls on graphite mimic DNA, Ultramicroscopy 42-44 (1992) 10731078.

[22] Chang H. and Bard A.J., Observation and characterization by scanning tunneling microscopy of structures generated by cleaving highly oriented pyrolytic graphite, Langmuir 7 (1991) 1143-1153.

[23] Stellwagen N.C., Anomalous electrophoresis of deoxyribonucleic acid restriction fragments on polyacrylamide gels, Biochemistry 22 (1983) 6186-6192.

[24] Martinez-Balbas A., Rodriguez-Campos A., Garcia-Ramirez M., Sainz J., Carrera P., Aymami J. and Azorin F., Satellite DNAs contain sequences that induce curvature, Biochemistry 29 (1990) 23422348.

[25] Muzard G., Théveny B. \& Révet B., Electron microscopy mapping of pBR322 DNA curvature. Comparison with theoretical models, EMBO J. 9 (1990) 1289-1298.

[26] Griffith J., DNA structure: evidence from electron microscopy, Science 201 (1978) 525-527.

[27] Shaiu W.-L., Larson D.D., Vesenka J. and Henderson E., Atomic force microscopy of oriented linear DNA molecules labeled with 5nm gold spheres, Nucleic Acids Res. 21 (1993) 99-103.

[28] Lindsay S.M. \& Philipp M., Can the scanning tunneling microscope sequence DNA? GATA 8 (1991) 8-13.

[29] Ulanovsky L. \& Trifonov E.N., Estimation of wedge components in curved DNA, Nature 326 (1987) 720-722.

[30] Kornberg A. \& Baker T.A., DNA replication (2nd edition. Freeman, New York, 1992).

[31] Lindsay S.M., Thundat T., Nagahara L., Knipping U. \& Rill R.L., Images of the DNA double helix in water, Science 244 (1989) 1063-1064. 
[32] Conner B.N., Takano T., Tanaka S., Itakura K. \& Dickerson R.E., The molecular structure of d(CpCpGpG) a fragment of right-handed double helical A-DNA, Nature 295 (1981) 294-299.

[33] Drew H.R., Wing R.M., Takano T., Broka C., Tanaka S., Itakura K. \& Dickerson R.E., Structure of a B-DNA dodecamer: conformation and dynamics, Proc. Natl. Acad. Sci. USA 78 (1981) 2179. 